\section{Refining the Human Behavior Small Group Experience}

To the Editor: Small group teaching is integral to our first-year Human Behavior course. To improve this experience, we made six fundamental changes in response to student feedback: relocating activities to clinical facilities, reducing group size, rotating to different clinical sites, revamping interviews to a rolling style, rotating faculty interviewers, and reassigning one faculty slot to a fixed senior psychiatry resident. By sharing our experiences thus far, we hope to inform others who are designing or restructuring their courses.

Prior to 2004-2005, the small group experience consisted of six interview sessions, occurring in various classrooms in the medical school. The class of approximately 120 students was divided into six groups of 20 students randomly assigned to two faculty group leaders. Each week, patients were recruited for the interview sessions largely from outpatient clinics and VA or hospital inpatient units. Given the logistics involved with outpatient participant recruitment, each week there were no-shows. Getting patients to commit to a 45-minute session with 20 students and two faculty members was not easy. The classroom setting also prohibited interviewing more acute patients who could not leave inpatient settings for safety reasons. The resulting scramble for interviewees often failed and faculty role play was substituted, leading to largely negative student feedback. Similarly, students complained about the "small" group size and the fact that faculty sometimes had emergencies on their units and were unable to attend, leaving just one discussant for 20 learners.

In spring 2005, small groups were relocated from classrooms to various inpatient and outpatient settings. This provided immediate access to a wealth of psychopathology and patient presentations and contextual relevance to the interviews.

To accommodate the smaller rooms available at the sites, the class was divided into nine groups (13 students per group). This reduction likely fostered greater interactions and more useful feedback from leaders. In contrast to previous years, most faculty members were now based in their own inpatient units or clinics and assigned to interview patients who corresponded with their areas of expertise. Over the 6 weeks, each group rotated to various sites to participate in sessions with different faculty for child, adolescent, and geriatric patients and patients with psy- chotic, anxiety, mood, and substance use disorders. The faculty's interview was limited to 10-15 minutes, subsequently opening up the session to student questions in a rolling interview style.

We also assigned senior residents as small group leaders who remained with the same group throughout the semester, allowing for greater continuity. The residents embraced the teaching opportunity and brought enthusiasm and the ability to relate with students generationally.

The change to in vivo groups and to residents as small group leaders greatly improved patient and instructor compliance with the sessions and also afforded exposures to more off-site faculty, who had been unable to participate previously for logistical reasons. Residents perceived the experience as rewarding, and the additional teaching responsibility motivated them to master diagnostics at a new level.

The changes may have played a role in improving the overall ratings of the small group experience from 4.3 ( $1=$ poor, $2=$ fair, $3=$ good, $4=$ very good, $5=$ excellent $)$ in 2004 to 4.5 in 2005 and 2006 and the overall ratings of the course from 4.0 in 2004 to 4.8 in 2005 and 4.7 in 2006.

Student feedback has been very positive. Students embraced working with and learning from residents, who often received high ratings. The interaction with residents also seemed to dispel some misconceptions about the type of individuals who go into psychiatry. Students enjoyed learning from a larger pool of faculty with a wide range of expertise.

We will continue to monitor feedback and make appropriate changes. We will also look more closely at resident perceptions and determine the effects these early experiences may have on their career trajectories (e.g., do they go on to academic careers?). One resident has won a prestigious national teaching fellowship and other local awards for which excellent evaluations as a Human Behavior small group leader may have been a contributing factor.

Robert N. Averbuch, M.D.

Christopher S. Wadsworth, M.D.

Jacqueline A. Hobbs, M.D., Ph.D.

Department of Psychiatry at University of Florida College of Medicine in Gainesville, FL

At the time of submission, the authors reported no competing interests. 